\title{
MicroRNA-26b is upregulated in a double transgenic mouse model of Alzheimer's disease and promotes the expression of amyloid- $\beta$ by targeting insulin-like growth factor 1
}

\author{
HONG LIU ${ }^{1 *}$, WENZHENG CHU ${ }^{1 *}$, LI GONG $^{1}$, XIAOYU GAO ${ }^{1}$ and WEI WANG ${ }^{2}$ \\ Departments of ${ }^{1}$ Neurology and ${ }^{2}$ Pharmacy, Yantai Yuhuangding Hospital, Yantai, Shandong 264000, P.R. China
}

Received February 6, 2015; Accepted December 18, 2015

DOI: $10.3892 / \mathrm{mmr} .2016 .4860$

\begin{abstract}
Alzheimer's disease (AD) is the most common form of dementia among the aging population. It is pathologically characterized by synaptic impairment, accumulation of neurofibrillary tangles and amyloid- $\beta$ (A $\beta$ ) deposition. MicroRNA-26b (miR-26b) has been observed to be upregulated in the human temporal cortex in $\mathrm{AD}$, however, the function of miR-26b has not been verified. Reverse transcription-quantitative polymerase chain reaction was conducted to investigate the expression levels of miR-26b in a double transgenic mouse model of AD. Following transfection of miR-26b or an miR-26b inhibitor, western blot analysis, enzyme-linked immunosorbent assay and luciferase assays were performed. The present study demonstrated that the expression levels of miR-26b were upregulated in a double transgenic mouse model of $\mathrm{AD}$. It was also demonstrated that upregulation of miR-26b in N2a/APP cells downregulated the insulin-like growth factor 1 (IGF-1) protein expression level and promoted $\mathrm{A} \beta$ production, whereas inhibition of miR-26b in N2a/APP cells upregulated the IGF-1 protein level and suppressed $A \beta$ production. Furthermore, miR-26b target sites in IGF-1 were confirmed using a luciferase assay in HEK293 cells. The present study may be useful in the development of effective therapeutic strategies against AD.
\end{abstract}

\section{Introduction}

Alzheimer's disease (AD), a progressive neurodegenerative disorder, is the most common form of dementia among the aging population (1). More than 3.5 million individuals worldwide have been diagnosed with $\mathrm{AD}$ and the proportion of

Correspondence to: Dr Wei Wang, Department of Pharmacy, Yantai Yuhuangding Hospital, 20 Yuhuangding East Road, Yantai, Shandong 264000, P.R. China

E-mail: doctor_weiwang@163.com

*Contributed equally

Key words: Alzheimer's disease, insulin-like growth factor-1, miR-26b, amyloid- $\beta$ people being diagnosed with $\mathrm{AD}$ after 85 years of age exceeds 1 in 3 (2). AD is clinically characterized by cognitive impairment leading to dementia, immobility, and eventually mortality (usually within a decade following the initial diagnosis) (3). The histopathological changes that occur in AD include widespread loss of neurons, and formation of senile plaques and neurofibrillary tangles (4). The predominant theory for the molecular mechanism underlying AD is the amyloid cascade hypothesis (5). Extracellular aberrant generation and/or inadequate clearance of amyloid- $\beta(A \beta)$, which originates from the amyloid precursor protein (APP), are considered to be responsible for the death of neurons and dementia in AD. An increased level of APP may increase the risk of AD (6-8). However, the underlying mechanisms of neurodegeneration, and the molecular and pathological components of the disease remain to be elucidated (9).

A number of microRNAs (miRs) have been implicated in $\mathrm{AD}$ (10-12). miRs are endogenous and evolutionarily conserved non-coding small RNA molecules (length, 21-25 nt). They form partially complementary base pairs within the 3'-untranslated regions (UTR) of protein-encoding mRNAs, resulting in the degradation of target transcripts or inhibition of translation $(13,14)$. miRs have been observed in various biological processes, including embryogenesis, the immune response, developmental timing, differentiation and organogenesis, cell-cycle control, proliferation and apoptosis (15-20). Multiple miRs have been observed to be aberrantly expressed in patients with AD (21). The dysregulation of miRs may participate in the progression of AD by influencing the expression and functions of their targets (22). For example, miR-339-5p and miR-153 are significantly downregulated in AD specimens, and may inhibit the expression of APP and BACE1. Thus, the identification of the targets of miRs is critical to understand the function of miRs in the development and progression of $\mathrm{AD}$. It also suggested that miRs may provide a target for therapeutic strategies for AD.

miR-26b was observed to be significantly upregulated in the human temporal cortex in AD (23); however, the function of miR-26b has not been verified. In the present study, miR-26b was upregulated in a double transgenic mouse model of AD. It was also demonstrated that upregulation of miR-26b in N2a/APP cells downregulated the insulin-like growth factor 1 (IGF-1) protein level and promoted $\mathrm{A} \beta$ production, whereas inhibition of miR-26b in N2a/APP cells upregulated the IGF-1 
protein level and suppressed $A \beta$ production. Furthermore, miR-26b target sites in IGF-1 were confirmed by a luciferase assay in HEK293 cells. The results of the present study may aid in the development of effective therapeutic strategies against AD.

\section{Materials and methods}

Transgenic mice and sacrifice. A total of nine APP/PS1 double-transgenic mice, aged 3, 6 or 9-months-old, and nine age-matched controls, were purchased from the Model Animal Research Center of Nanjing University (Nanjing, China), originally obtained from The Jackson Laboratory (Bar Harbor, ME, USA). They were maintained at $19-23^{\circ} \mathrm{C}$ under a 12-h light/dark cycle with ad libitum access to sterile food and water, and all animal handling was conducted in accordance with institutional guidelines. The present study was approved by the ethics committee of Yantai Yuhuangding Hospital (Yantai, China).

The mice were sacrificed by intraperitoneal injection with pentobarbital overdose $(50 \mathrm{mg} / \mathrm{kg}$; Beyotime Institute of Biotechnology, Haimen, China), followed by the removal and dissection of the brain tissues. The cortexes of the brains were frozen in liquid nitrogen for further RNA extraction.

Cell culture and transfection. The HEK293 human embryonic kidney cell line was purchased from the Shanghai Institute of Biochemistry and Cell Biology (Shanghai, China). N2a/WT and N2a/APP cells were a gift from the Tianjin Medical University (Tianjin, China). HEK293 cells were cultured in Opti-MEM medium (Gibco; Thermo Fisher Scientific, Inc., Waltham, MA, USA) supplemented with $10 \%$ fetal bovine serum (FBS; Gibco; Thermo Fisher Scientific, Inc.). N2a/WT and N2a/APP cells were cultured in medium containing 50\% Dulbecco's modified Eagle's medium (Gibco; Thermo Fisher Scientific, Inc.), and 45\% Opti-MEM supplemented with 5\% FBS. All cell lines were incubated in a humidified air atmosphere of $5 \% \mathrm{CO}_{2}$ at $37^{\circ} \mathrm{C}$.

Cells were transfected with miR-26b mimic, negative control (NC), miR-26b inhibitor or NC inhibitor (Shanghai GenePharma, Co., Ltd., Shanghai, China), at a final concentration of $50 \mathrm{nM}$, using Lipofectamine 2000 (Invitrogen; Thermo Fisher Scientific, Inc.) according to the manufacturer's protocols.

Reverse transcription-quantitative polymerase chain reaction $(R T-q P C R)$. Total RNA was extracted from tissues using mirVana miRNA Isolation kit (Ambion; Thermo Fisher Scientific, Inc.) and DNase I (Ambion; Thermo Fisher Scientific, Inc.), and treated according to the manufacturer's protocol to obtain DNA-free RNA. Equal quantities of RNA were subjected to cDNA synthesis using the miScript Reverse Transcription kit (Qiagen, Inc., Valencia, CA, USA). RT-qPCR for miR-26b was performed in a Lightcycler (Roche Diagnostics GmbH, Mannheim, Germany), according to the SYBR Green detection protocol, and all reactions were run in triplicate. Each reaction was performed in a final volume of $20 \mu \mathrm{l}$. The PCR cycling conditions were as follows: $95^{\circ} \mathrm{C}$ for $15 \mathrm{~min}$, followed by 40 cycles of $94^{\circ} \mathrm{C}$ for $15 \mathrm{sec}, 55^{\circ} \mathrm{C}$ for $30 \mathrm{sec}$ and $70^{\circ} \mathrm{C}$ for $30 \mathrm{sec}$. Primers for mature miR-26b and U6 snRNA were purchased from Qiagen, Inc. Expression was determined in 18 mice (six APP/PS1 mice and six control mice). Relative expression levels were calculated using the $2^{-\Delta \Delta C \mathrm{C}}$ method (24). Every sample was replicated three times.

Western blot analysis. Cells were washed with ice-cold phosphate-buffered saline (PBS) and solubilized in cold radioimmunoprecipitation lysis buffer (RIPA; Beyotime Institute of Biotechnology) $72 \mathrm{~h}$ after transfection. Cells were incubated at $0^{\circ} \mathrm{C}$ for $15 \mathrm{~min}$ and centrifuged at $2,000 \mathrm{x}$ g for $10 \mathrm{~min}$ at $4{ }^{\circ} \mathrm{C}$. The supernatants were collected, and the protein concentration was measured using a bicinchoninic acid protein assay kit (Beyotime Institute of Biotechnology). Samples were boiled for 5 min in loading buffer (Beyotime Institute of Biotechnology) and then equal quantities of the proteins $(40 \mu \mathrm{g})$ were separated by $10 \%$ sodium dodecyl sulfate-polyacrylamide gel electrophoresis (Beyotime Institute of Biotechnology) and then transferred to a polyvinylidene difluoride membrane (Beyotime Institute of Biotechnology). The membrane was blocked with 5\% non-fat dry milk for $2 \mathrm{~h}$, followed by an overnight incubation at $4{ }^{\circ} \mathrm{C}$ with primary mouse anti-human IGF-1 (1:1,000; sc-74116; Santa Cruz Biotechnology, Inc., Dallas, TX, USA) and mouse anti-human $\beta$-actin (1:1,000; sc-130301; Santa Cruz Biotechnology, Inc.) monoclonal antibodies. Following washing with PBS three times (for 5 min each time), the membrane was incubated for $1 \mathrm{~h}$ at room temperature with goat anti-mouse horseradish peroxidase (HRP)-conjugated secondary antibody (1:500; sc-2005; Santa Cruz Biotechnology, Inc.) in Tris-buffered saline with Tween 20 (Beyotime Institute of Biotechnology). The blot was detected with an ECL kit (Pierce Biotechnology, Inc., Rockford, IL, USA) and images were captured using a FluorChem imaging system (ProteinSimple, San Jose, CA, USA). The protein intensities were quantified using the AlphaEaseFC 4.1.0 software (Alpha Innotech, San Leandro, CA, USA).

Enzyme-linked immunosorbent (ELISA) assay. A 342 levels in cell lysates were quantified using a mouse ELISA assay according to the manufacturer's protocols (Abcam, Cambridge, UK), as described previously (25). A $\beta 42$ in samples was captured with G2-11, a monoclonal antibody specific for $\mathrm{A} \beta 42$ (Abeta $\mathrm{GmbH}$, Heidelberg, Germany). A $\beta 42$ was then probed specifically with the antibody Biotin-Wo2 (Abeta $\mathrm{GmbH}$ ) overnight at $4^{\circ} \mathrm{C}$, and finally developed with NeutrAvidin-HRP (Pierce Biotechnology, Inc.). The HRP activity was measured with the TMP Microwell Peroxidase system (KPL, Inc., Gaithersburg, MD, USA).

Target Prediction of miR-26b. TargetScan 5.2 (http://www. targetscan.org/) was used to predict the target genes of miR-26b.

Luciferase assay. The luciferase reporter plasmid and the wild-type (WT)-pGL3-IGF-1-3'UTR Wt and mutant (Mut)-pGL3-IGF-1-3'UTR expression vectors, were obtained from Shanghai GenePharma. The HEK293 human embryonic kidney cells were plated at $\sim 90 \%$ confluence and transfected with the reporter plasmid, miR-26b mimics or $\mathrm{NC}$ in a 12-well plate using Lipofectamine 2000, according to the manufacturer's protocol. The Renilla and the firefly luciferase activity were measured following 48-h incubation using the Dual-Luciferase Reporter assay system (Promega Corporation, Madison, 


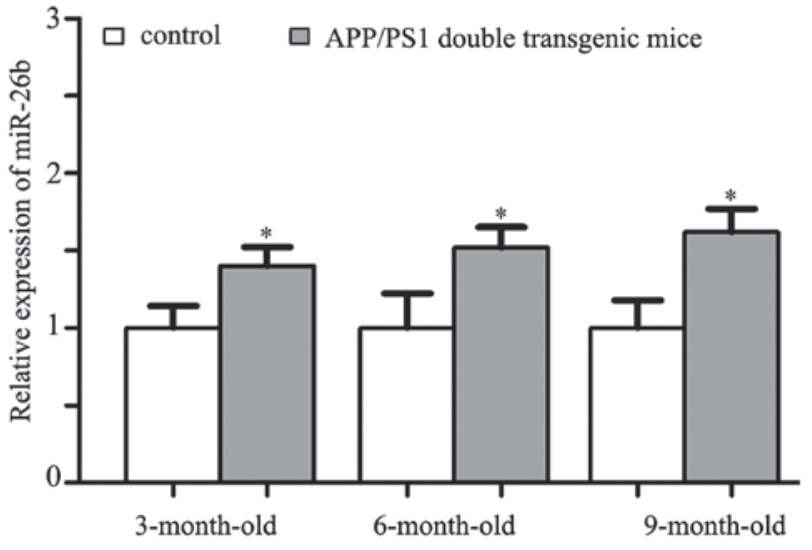

Figure 1. Expression of miR-26b in APP/PS1 double-transgenic mice and age-matched controls. miR-26b was increased in 3,6 and 9-month-old APP/PS1 double-transgenic mice compared with age-matched controls. Data are presented as the mean \pm standard deviation. ${ }^{*} \mathrm{P}<0.05$ vs. the control. miR-26b, microRNA-26b.

A
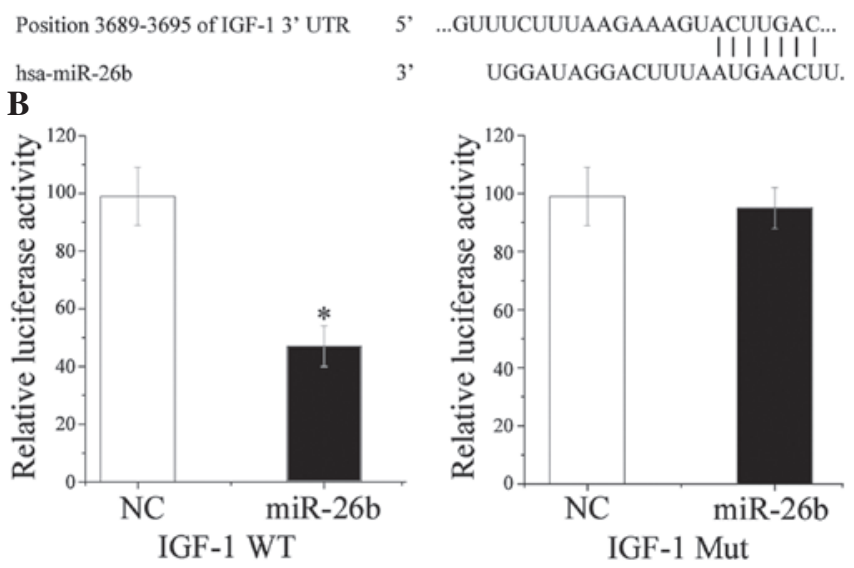

Figure 2. (A) TargetScan 5.2 determined that IGF-1 mRNA contained a nmiR-26b seven-nucleotide seed match at position 3689-3695 of the IGF-1 3'-UTR. (B) IGF-1 is a direct target of miR-26b in vitro. Overexpression of miR-26b suppressed IGF-1 3' UTR-luciferase activity by $48 \%$ in HEK293 cells. Data are presented as the mean \pm standard deviation. ${ }^{~} \mathrm{P}<0.05$ vs. the control. UTR, untranslated region; miR-26b, microRNA-26b; WT, wild-type; Mut, mutant; IGF-1, insulin-like growth factor 1.

WI, USA). The firefly and Renilla luciferase activities were measured with a luminometer (Tecan Group, Ltd., Männedorf, Switzerland). The firefly luciferase activity was normalized to Renilla luciferase activity for each transfected well. Each assay was replicated three times.

Statistical analysis. Data are presented as the mean \pm standard deviation and compared using Student's t-test in Stata, version 10.0 (StataCorp LP, College Station, TX, USA). P<0.05 was considered to indicate a statistically significant difference.

\section{Results}

Expression of miR-26b in APP/PS1 double-transgenic mice. In previous miR profiling experiments, $\mathrm{miR}-26 \mathrm{~b}$ was observed to be upregulated in human brains from patients with $\mathrm{AD}(23)$. In the present study, APP/PS1 double-transgenic mice and
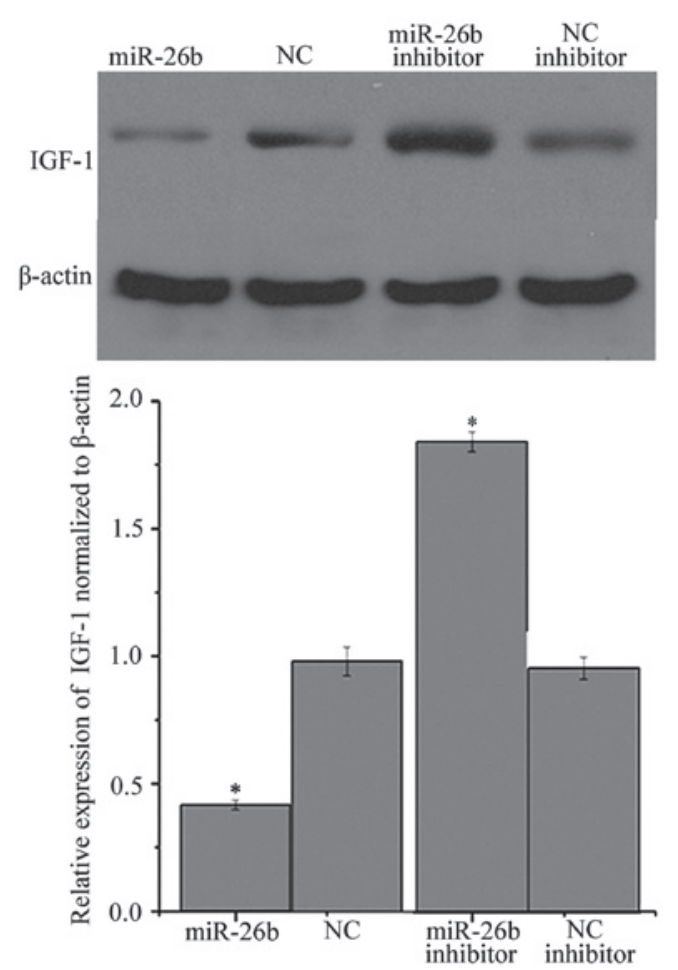

Figure 3. IGF-1 protein expression levels were significantly downregulated, as compared with that in N2a/WT cells transfected with NC. By contrast, treatment with the miR-26b inhibitor led to a significant increase in the expression level of the IGF-1 protein compared with that of the N2a/WT cells transfected with an NC inhibitor. Data are presented as the mean \pm standard deviation. ${ }^{*} \mathrm{P}<0.05$ vs. the control. NC, negative control; miR-26b, microRNA-26b; WT, wild-type; IGF-1, insulin-like growth factor 1.

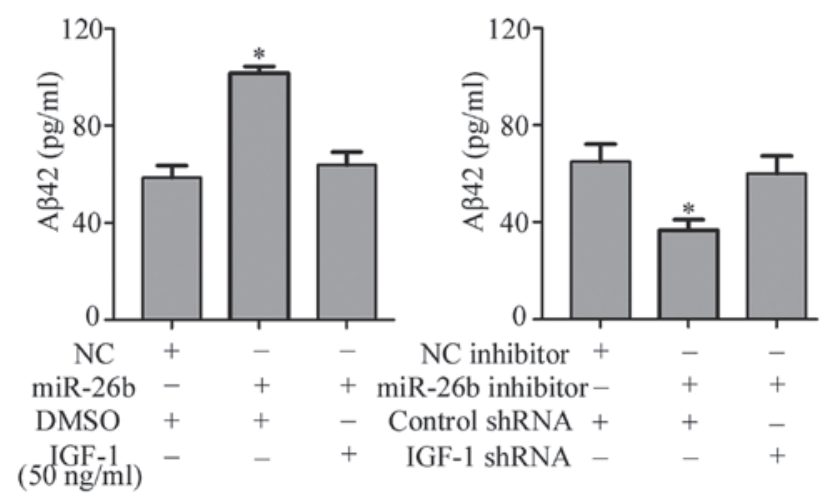

Figure 4. miR-26b increases A $\beta 42$ production by targeting IGF-1. The level of $\mathrm{A} \beta 42$ in N2a/APP cell lysates was significantly increased following transfection with miR-26b, whereas $50 \mathrm{ng} / \mathrm{ml}$ IGF-1 supplementation reversed this upregulation. By contrast, the level of A 342 in the lysates of N2a/APP was downregulated following transfection with an miR-26b inhibitor. Data are presented as the mean \pm standard deviation. ${ }^{*} \mathrm{P}<0.05$ vs. the control. $\mathrm{NC}$, negative control; DMSO, dimethyl sulfoxide; IGF-1, insulin-like growth factor 1; miR-26b, microRNA-26b.

age-matched controls were used to investigate the expression levels of miR-26b by RT-qPCR. Results of the present study demonstrated that the expression levels of miR-26b were increased in 3,6 and 9-month-old APP/PS1 double-transgenic mice compared with the age-matched controls (Fig. 1). The results indicated that miR-26b was upregulated in APP/PS1 double-transgenic mice. 
$I G F-1$ is a direct target of $m i R-26 b$. In a previous study, the protein expression level of IGF-1 was significantly lower in APP/PS1 double-transgenic mice, as compared with control mice; however, no significant difference was observed in the mRNA expression level of IGF-1 between the APP/PS1 double-transgenic and control mice (23). These findings suggested that the regulation of IGF-1 expression occurred at the post-transcriptional level in APP/PS1 mice. In order to further confirm the association between IGF-1 and its regulators in APP/PS1 mice, as compared with control mice, the present study used TargetScan 5.2 to assess the complementarity of miR-26b to the IGF-1 3'-UTR. As presented in Fig. 2A, one binding site of miR-26b was observed within the 3'-UTR of IGF-1. In addition, luciferase reporter assays were performed to investigate whether IGF-1 is a direct target of miR-26b. As presented in Fig. 2B, the luciferase activity was significantly inhibited in cells co-transfected with miR-26b and WT-3'-UTR compared with the control vector group, whereas Mut-3'-UTR luciferase activity changed only marginally, this suggests that IGF-1 may be a direct target of miR-26b in vitro.

miR-26b reduces the level of IGF-1. To directly investigate whether miR-26b reduces the expression of IGF-1, western blot analysis was performed. As presented in Fig. 3, the IGF-1 protein level was significantly downregulated compared with that in $\mathrm{N} 2 \mathrm{a} / \mathrm{WT}$ cells transfected with the NC. By contrast, treatment with the miR-26b inhibitor led to a significant increase in the level of IGF-1 protein compared with that in $\mathrm{N} 2 \mathrm{a} / \mathrm{WT}$ cells transfected with NC inhibitor.

miR-26b increases $A \beta$ production by targeting IGF-1. To investigate the role of miR-26b in AD, ELISA analysis was performed to observe the level of $A \beta 42$ in lysates of N2a/APP cells. As presented in Fig. 4, the level of A $\beta 42$ in N2a/APP cell lysates was significantly increased following transfection with miR-26b, whereas addition of $50 \mathrm{ng} / \mathrm{ml} \mathrm{IGF-1} \mathrm{supple-}$ mentation reversed this upregulation. By contrast, the level of $A \beta 42$ in the lysates of N2a/APP cells was downregulated following transfection with an miR-26b inhibitor. These data indicate that miR-26b negatively regulates IGF-1 translation and induces $A \beta$ production in vitro.

\section{Discussion}

Previous studies have demonstrated that miR-26b is frequently downregulated in various tumors, including breast cancer (26), nasopharyngeal carcinoma (27), hepatocellular carcinoma (28), squamous cell carcinoma of the tongue (29), primary squamous cell lung carcinoma (30) and squamous cell carcinoma of glioma (31). It has been reported to be a critical regulator in carcinogenesis and tumor progression by acting as a tumor suppressor gene in various types of cancer $(27,31,32)$. miR-26 has been observed to be upregulated in the human temporal cortex in AD (23). In the present study, miR-26b was observed to be upregulated in APP/PS1 double-transgenic mice, suggesting that miR-26b may function in the development of AD.

The potential benefit of the analysis of miRs in the diagnosis and treatment of numerous diseases, including cancer, infection and neurodegenerative disease, has been previously evaluated in numerous studies $(9,11,33)$. The expression of miRs is known to be altered in multiple regions of the brain in $\mathrm{AD}$, however, the cause and consequence in the pathology of the disease remains to be elucidated (8). Downregulation of IGF-1 associated with accelerated accumulation of $A \beta$ in the brain is a feature of AD (34). In the present study, it was demonstrated that upregulation of miR-26b in N2a/APP cells downregulated the IGF-1 protein level and promoted $\mathrm{A} \beta$ production, whereas inhibition of miR-26b in N2a/APP cells upregulated the IGF-1 protein level and suppressed $A \beta$ production. Furthermore, miR-26b target sites in IGF-1 were confirmed by a luciferase assay in HEK 293 cells. These results suggested that miR-26b may be considered a novel therapy for patients with $\mathrm{AD}$.

$\mathrm{AD}$ is the most common form of dementia in the elderly. It is pathologically characterized by synaptic impairment, accumulation of neurofibrillary tangles, and $\mathrm{A} \beta$ deposition (35). IGF-1 is a member of the insulin family of hormones (36), and-1 is part of an evolutionarily conserved signaling pathway. It is involved in neuronal growth, survival and differentiation, and it promotes neurite outgrowth, migration, protein synthesis, neuronal cytoskeletal protein expression, and nascent synapse formation (37-41). Previous studies have demonstrated the role of IGF-1 signaling in AD-pathogenesis using various animal and human models of AD (42-44); the serum IGF-1 levels were significantly downregulated in patients with $\mathrm{AD}$, as compared with patients with vascular dementia or age-matched non-demented elderly subjects $(43,44)$. IGF-1 may contribute to the regulation of $\tau$ phosphorylation, amyloid precursor protein (APP) cleavage, $\mathrm{A} \beta$ transport and degradation, memory formation, aging and longevity in AD (45).

The major neuropathological finding in $\mathrm{AD}$ is considered to be the presence of high levels of $A \beta$ in the brain samples. These peptides are neurotoxic and form amyloid plaques (46). The association between serum IGF-1 and brain amyloidosis was established by a previous study indicating a potential role of IGF-1 in the clearance of A $\beta$ from the brain (34). Systemic IGF-1 administration proved effective in lowering brain $A \beta$ levels, while blockade of systemic IGF-1 action was sufficient to induce brain amyloidosis $(34,47)$. Furthermore, reduced IGF-1 input lowers neuronal resistance to $A \beta$ peptide toxicity, increases cellular susceptibility to cell death signals and leads, ultimately, to the accumulation of $\mathrm{A} \beta$ in the brain (48). The findings of these previous studies suggest that IGF-1 is a potential therapeutic target in AD. Upregulation of IGF-1 may, therefore, provide neuroprotection, facilitate $A \beta$ clearance, antagonize the deleterious effects of tumor necrosis factor- $\alpha$ and inhibit certain features of the inflammatory reaction (49). miR-based therapy is expected to be more efficient than the traditional single target therapy, since miRs regulate multiple target genes simultaneously (50). The results of the present study may aid the development effective therapeutic strategies against AD.

In conclusion, this is the first study to demonstrate that miR-26b was downregulated in APP/PS1 double-transgenic mice and negatively regulated the expression of IGF-1 in vitro. As IGF-1 is critical in A $\beta$ formation, increasing IGF-1 expression levels is suggested as a potential therapeutic strategy for AD. Results of the present study suggest that miR-26b is 
a potential therapeutic target for the downregulation of $A \beta$ formation. However, further studies in vivo are required to address the delivery of the miR-26b inhibitor into the mouse hippocampus using adenoviruses to determine whether this upregulates IGF-1 protein levels and reduces $\mathrm{A} \beta$ formation.

\section{References}

1. Wang LL, Huang Y, Wang G and Chen SD: The potential role of microRNA-146 in Alzheimer's disease: Biomarker or therapeutic target? Med Hypotheses 78: 398-401, 2012.

2. Alzheimer's Association: 2013 Alzheimer's disease facts and figures. Alzheimers Dement 9: 208-245, 2013.

3. Banzhaf-Strathmann J, Benito E, May S, Arzberger T, Tahirovic S, Kretzschmar H, Fischer A and Edbauer D: MicroRNA-125b induces tau hyperphosphorylation and cognitive deficits in Alzheimer's disease. EMBO J 33: 1667-1680, 2014.

4. Giannakopoulos P, Hof PR and Bouras C: Selective vulnerability of neocortical association areas in Alzheimer's disease. Microsc Res Tech 43: 16-23, 1998.

5. Karran E, Mercken M and De Strooper B: The amyloid cascade hypothesis for Alzheimer's disease: An appraisal for the development of therapeutics. Nat Rev Drug Discov 10: 698-712, 2011.

6. Lewczuk P, Kamrowski-Kruck H, Peters O, Heuser I, Jessen F, Popp J, Bürger K, Hampel H, Frölich L, Wolf S, et al: Soluble amyloid precursor proteins in the cerebrospinal fluid as nove potential biomarkers of Alzheimer's disease: A multicenter study. Mol Psychiatry 15: 138-145, 2010.

7. Weiner MW: Dementia in 2012: Further insights into Alzheimer disease pathogenesis. Nat Rev Neurol 9: 65-66, 2013.

8. Liu CG, Wang JL, Li L and Wang PC: MicroRNA-384 regulates both amyloid precursor protein and $\beta$-secretase expression and is a potential biomarker for Alzheimer's disease. Int J Mol Med 34: 160-166, 2014.

9. Liu CG, Song J, Zhang YQ and Wang PC: MicroRNA-193b is a regulator of amyloid precursor protein in the blood and cerebrospinal fluid derived exosomal microRNA-193b is a biomarker of Alzheimer's disease. Mol Med Rep 10: 2395-2400, 2014.

10. Chan AW and Kocerha J: The path to microRNA therapeutics in psychiatric and neurodegenerative disorders. Front Genet 3: 82, 2012.

11. Delay C, Mandemakers W and Hébert SS: MicroRNAs in Alzheimer's disease. Neurobiol Dis 46: 285-290, 2012.

12. Delay C and Hébert SS: MicroRNAs and Alzheimer's disease mouse models: Current insights and future research avenues. Int J Alzheimers Dis 2011: 894938, 2011.

13. Lee RC, Feinbaum RL and Ambros V: The C. elegans heterochronic gene lin-4 encodes small RNAs with antisense complementarity to lin-14. Cell 75: 843-854, 1993.

14. Wang SE and Lin RJ: MicroRNA and HER2-overexpressing cancer. Microrna 2: 137-147, 2013.

15. Alvarez-Garcia I and Miska EA: MicroRNA functions in animal development and human disease. Development 132: 4653-4662, 2005.

16. Decembrini S, Bressan D, Vignali R, Pitto L, Mariotti S, Rainaldi G, Wang X, Evangelista M, Barsacchi G and Cremisi F: MicroRNAs couple cell fate and developmental timing in retina. Proc Natl Acad Sci USA 106: 21179-21184, 2009.

17. Rosa A and Brivanlou AH: MicroRNAs in early vertebrate development. Cell Cycle 8: 3513-3520, 2009.

18. Lu LF and Liston A: MicroRNA in the immune system, microRNA as an immune system. Immunology 127: 291-298, 2009.

19. Trang P, Weidhaas JB and Slack FJ: MicroRNAs as potential cancer therapeutics. Oncogene 27 (Suppl 2): S52-S57, 2008.

20. Bartel DP: MicroRNAs: Genomics, biogenesis, mechanism and function. Cell 116: 281-297, 2004.

21. Wang WX, Huang Q, Hu Y, Stromberg AJ and Nelson PT: Patterns of microRNA expression in normal and early Alzheimer's disease human temporal cortex: White matter versus gray matter. Acta Neuropathol 121: 193-205, 2011.

22. Eacker SM, Dawson TM and Dawson VL: Understanding microRNAs in neurodegeneration. Nat Rev Neurosci 10: 837-841, 2009.

23. Absalon S, Kochanek DM, Raghavan V and Krichevsky AM: MiR-26b, upregulated in Alzheimer's disease, activates cell cycle entry, tau-phosphorylation and apoptosis in postmitotic neurons. J Neurosci 33: 14645-14659, 2013.
24. Livak KJ and Schmittgen TD: Analysis of relative gene expression data using real-time quantitative PCR and the 2- $\Delta \Delta \mathrm{Ct}$ method. Methods 25: 402-408, 2001.

25. Zhang YC, Wang ZF, Wang Q, Wang YP and Wang JZ: Melatonin attenuates beta-amyloid-induced inhibition of neurofilament expression. Acta Pharmacol Sin 25: 447-451, 2004.

26. Li J, Kong X, Zhang J, Luo Q, Li X and Fang L: MiRNA-26b inhibits proliferation by targeting PTGS2 in breast cancer. Cancer Cell Int 13: 7, 2013.

27. Ji Y, He Y, Liu L and Chong X: MiRNA-26b regulates the expression of cyclooxygenase-2 in desferrioxamine-treated CNE cells. FEBS Lett 584: 961-967, 2010.

28. Ji J, Shi J, Budhu A, Yu Z, Forgues M, Roessler S, Ambs S, Chen Y, Meltzer PS, Croce CM, et al: MicroRNA expression, survival and response to interferon in liver cancer. N Engl J Med 361: 1437-1447, 2009.

29. Cao J, Guo T, Dong Q, Zhang J and Li Y: miR-26b is downregulated in human tongue squamous cell carcinoma and regulates cell proliferation and metastasis through a COX-2-dependent mechanism. Oncol Rep 33: 974-980, 2015.

30. Gao W, Shen H, Liu L, Xu J, Xu J and Shu Y: MiR-21 overexpression in human primary squamous cell lung carcinoma is associated with poor patient prognosis. J Cancer Res Clin Oncol 137: 557-566, 2011

31. Wu N, Zhao X, Liu M, Liu H, Yao W, Zhang Y, Cao S and Lin X: Role of microRNA-26b in glioma development and its mediated regulation on EphA2. PLoS One 6: e16264, 2011.

32. Li J, Li X, Kong X, Luo Q, Zhang J and Fang L: MiRNA-26b inhibits cellular proliferation by targeting CDK8 in breast cancer. Int J Clin Exp Med 7: 558-565, 2014.

33. Dassow $\mathrm{H}$ and Aigner A: MicroRNAs (miRNAs) in colorectal cancer: From aberrant expression towards therapy. Curr Pharm Des 19: 1242-1252, 2013.

34. Carro E, Trejo JL, Gomez-Isla T, LeRoith D and Torres-Aleman I: Serum insulin-like growth factor I regulates brain amyloid-beta levels. Nat Med 8: 1390-1397, 2002.

35. Xiao AW, He J, Wang Q, Luo Y, Sun Y, Zhou YP, Guan Y, Lucassen PJ and Dai JP: The origin and development of plaques and phosphorylated tau are associated with axonopathy in Alzheimer's disease. Neurosci Bull 27: 287-299, 2011.

36. Jones JI and Clemmons DR: Insulin-like growth factors and their binding proteins: Biological actions. Endocr Rev 16: 3-34, 1995.

37. van Exel E, Eikelenboom P, Comijs H, Deeg DJ, Stek ML and Westendorp RG: Insulin-like growth factor-1 and risk of late-onset Alzheimer's disease: Findings from a family study. Neurobiol Aging 35: 725e7-e10, 2014.

38. Ye P, Xing Y, Dai Z and D'Ercole AJ: In vivo actions of insulin-like growth factor-I (IGF-I) on cerebellum development in transgenic mice: Evidence that IGF-I increases proliferation of granule cell progenitors. Brain Res Dev Brain Res 95: 44-54, 1996.

39. D'Ercole AJ, Ye P and O'Kusky JR: Mutant mouse models of insulin-like growth factor actions in the central nervous system. Neuropeptides 36: 209-220, 2002.

40. Popken GJ, Hodge RD, Ye P, Zhang J, Ng W, O'Kusky JR and D'Ercole AJ: In vivo effects of insulin-like growth factor-I (IGF-I) on prenatal and early postnatal development of the central nervous system. Eur J Neurosci 19: 2056-2068, 2004.

41. O'Kusky JR, Ye P and D'Ercole AJ: Insulin-like growth factor-I promotes neurogenesis and synaptogenesis in the hippocampal dentate gyrus during postnatal development. J Neurosci 20: 8435-8442, 2000.

42. Wang W, Yu JT, Tan L, Liu QY, Wang HF and Ma XY: Insulin-like growth factor 1 (IGF1) polymorphism is associated with Alzheimer's disease in Han Chinese. Neurosci Lett 531: 20-23, 2012.

43. Rollero A, Murialdo G, Fonzi S, Garrone S, Gianelli MV, Gazzerro E, Barreca A and Polleri A: Relationship between cognitive function, growth hormone and insulin-like growth factor I plasma levels in aged subjects. Neuropsychobiology 38: 73-79, 1998.

44. Watanabe T, Miyazaki A, Katagiri T, Yamamoto H, Idei T and Iguchi T: Relationship between serum insulin-like growth factor-1 levels and Alzheimer's disease and vascular dementia. J Geriatr Soc 53: 1748-1753, 2005.

45. Freude S, Schilbach K and Schubert M: The role of IGF-1 receptor and insulin receptor signaling for the pathogenesis of Alzheimer's disease: From model organisms to human disease. Curr Alzheimer Res 6: 213-223, 2009. 
46. Yankner BA, Dawes LR, Fisher S, Villa-Komaroff L, Oster-Granite ML and Neve RL: Neurotoxicity of a fragment of the amyloid precursor associated with Alzheimer's disease. Science 245: 417-420, 1989.

47. Carro E, Trejo JL, Gerber A, Loetscher H, Torrado J, Metzger F and Torres-Aleman I: Therapeutic actions of insulin-like growth factor I on APP/PS2 mice with severe brain amyloidosis. Neurobiol Aging 27: 1250-1257, 2006.
48. Carro E and Torres-Aleman I: Insulin-like growth factor I and Alzheimer's disease: Therapeutic prospects? Expert Rev Neurother 4: 79-86, 2004.

49. Gasparini $\mathrm{L}$ and $\mathrm{Xu} \mathrm{H}$ : Potential roles of insulin and IGF-1 in Alzheimer's disease. Trends Neurosci 26: 404-406, 2003.

50. Daválos A and Suárez Y: MiRNA-based therapy: From bench to bedside. Pharmacol Res 75: 1-2, 2013. 